\title{
Effects of intradialytic aerobic exercise on hemodialysis patients: a systematic review and meta-analysis
}

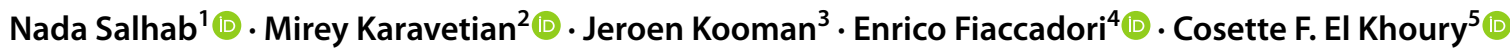

Received: 28 August 2018 / Accepted: 19 November 2018 / Published online: 18 January 2019

(c) The Author(s) 2019

\begin{abstract}
Objective Intradialytic exercise (IDE) is not yet a routine practice for hemodialysis patients, the lack of guidelines supporting it being a major reason. This systematic review and meta-analysis of aerobic IDE interventions examined the efficacy of IDE regarding quality of life (QOL), serum phosphorus, dialysis efficiency, inflammatory status, vitamin D3, parathyroid hormone, intake of phosphate binders, mortality and hospitalization rate.

Methods Pubmed, Medline (Ovid), Embase (Ovid), Cochrane, and Cinahl (EBSCO) databases were searched to retrieve studies up to June 12, 2018. A manual reference search was also performed. Studies were included if they evaluated (a) aerobic IDE effect on at least one of our study parameters, (b) adult hemodialysis patients, (c) patients for $>1$ month.

Results Twenty-two studies were retrieved (706 participants), of which 12 were eligible for meta-analysis. Aerobic IDE had a significant positive effect on the QOL physical component score (QOL-PSC) and on mental component score (QOL-MCS) of SF36, but not on serum phosphorus or Kt/V.

Conclusions IDE incorporation into clinical practice has a significant positive effect on QOL-PSC and QOL-MCS. In the reviewed studies, IDE did not result in any health hazard in hemodialysis patients. Nevertheless, future research should assess the long-term effectiveness and safety of IDE. The limitations of this review include the lack of quality analysis of the studies, the limited number of studies that could be included in the meta-analysis, the diversity in the exercise intensity, duration and modality, and the limited data for several outcomes.
\end{abstract}

Prospero registration ID CRD42016052062.

Keywords Hemodialysis $\cdot$ Intradialytic exercise $\cdot$ Quality of life $\cdot$ Phosphorus $\cdot$ Meta-analysis

Nada Salhab

n.salhab@maastrichtuniversity.nl

1 School of Nutrition and Translational Research in Metabolism, Faculty of Health Medicine and Life Sciences, Maastricht University, Maastricht, The Netherlands

2 Department of Health Sciences, College of Natural Health Sciences, Zayed University, Dubai, United Arab Emirates

3 Department of Internal Medicine, Division of Nephrology, University Hospital Maastricht, Maastricht, The Netherlands

4 Internal Medicine and Nephrology Department, Parma University Medical School, Parma, Italy

5 School of Public Health and Primary Care, Faculty of Health Medicine and Life Sciences, Maastricht University, Maastricht, The Netherlands

\section{Introduction}

Intradialytic exercise (IDE) is defined as exercise training performed during the hemodialysis (HD) session to increase the patient's strength and endurance, and hence targeting various physiological and psychosocial parameters. The nature of the IDE varies from resistance to aerobic exercise and stretching, with different equipment used corresponding to the type of exercise. IDE has demonstrated a positive effect on the overall health and hospitalization rate of HD patients $[1,2]$.

The Kidney Disease Improving Global Outcomes (KDIGO) guidelines recommend a full integration of exercise in the daily life of chronic kidney disease patients (at least $30 \mathrm{~min} /$ day, 5 times a week) taking into consideration their cardiovascular health and level of tolerance [3]. Unfortunately, there are no clear guidelines for IDE, and the IDE 
experience is described, from the patient's perspective, as "going into the unknown" [4].

Sedentary lifestyle, low quality of life and reduced $\mathrm{VO}_{2}$ max are associated with increased mortality risk among HD patients [5-7]. IDE has shown to have positive effects in HD patients; this was evident from the results of previous systematic reviews where IDE improved the efficiency of dialysis $(\mathrm{Kt} / \mathrm{V})[8,9], \mathrm{VO}_{2}$ max [8-10], and quality of life (QOL) [8-11]. In addition, several studies have also shown positive effects of IDE on solute clearance, notably with regard to serum phosphorus levels (P) [1, 12-14]. Possible explanations of the underlying mechanism are the increased cardiac output and blood flow to lower extremities, and capillary vasodilation, resulting in more solutes being transferred to the vascular compartment and reaching the dialyzer membrane for diffusion [15]. Thus IDE could be a cornerstone in the management of HD patients.

Capitanini et al. suggested that, to sustain an exercise program for HD patients, there needs to be a healthcare team led by a nephrologist and including a cardiologist, physiotherapist, exercise physiologist, renal dietitian, and a nurse [16]. To date, some dialysis units are lucky enough to have ergometers that connect to the HD beds or chairs so permitting their HD patients to benefit from this IDE aerobic exercise. Furthermore, exercise prescription for several chronic conditions [17] is in alignment with the current international physical activity (PA) guidelines which is $150 \mathrm{~min} /$ week of moderate intensity aerobic PA [18]. Even though other recommendations include resistance training, this review will focus on the effects of aerobic IDE since combined training is more complex for HD patients.

This review adds serum phosphate $(\mathrm{P})$ as a new parameter (with respect to existing reviews), since it is an important risk factor associated with negative outcomes in HD patients [19]. The objective of this review is to explore all published interventions on aerobic IDE, identify the optimal protocols used, and highlight the effects of these trials on patient QOL, P, dialysis efficiency (urea reduction ratio [URR], $\mathrm{Kt} / \mathrm{V}$ ), vitamin $\mathrm{D} 3$, parathyroid hormone (PTH), and serum $\mathrm{C}$-reactive protein (CRP), intake of $\mathrm{P}$ binders, the number of emergency HD sessions, cost effectiveness, mortality and hospitalization rate.

\section{Methods}

\section{Eligibility criteria}

All eligible studies were included if they met the predetermined inclusion and exclusion criteria detailed in the PICOS model (Table 1). There was no language restriction, nor filters applied to the search.
Table 1 Eligibility criteria

\begin{tabular}{lll}
\hline Criteria & Inclusion criteria & Exclusion criteria \\
\hline $\begin{array}{l}\text { Population } \\
\text { Intervention }\end{array}$ & Chronic adult HD patients & All other patients \\
& Aerobic IDE & Non aerobic exercise \\
Non-IDE intervention \\
Outcomes & Pre-post intervention & \\
& QOL & \\
& Serum P & \\
& Kt/v & \\
& URR & \\
& Vitamin D3 & \\
& PTH & \\
& CRP & \\
& Mortality & Interventions $<1$ month \\
Study types & Hospitalization rate & Non-interventional trials \\
& Interventional studies & Conference abstracts \\
& & Systematic reviews \\
& & Meta-analyses \\
\hline & & \\
& &
\end{tabular}

\section{Search strategy}

Potential studies were identified using the Pubmed, Medline (Ovid), Embase (Ovid), Cochrane, and Cinahl (EBSCO) databases up to June 12, 2018. In addition, references of included articles were hand-searched by 2 reviewers; pertinent references were retrieved and submittted to full text screening. The search strategy included the following terms selected from the Medical Subject Headings (MeSH): renal replacement therapy, renal dialysis, hemodiafiltration, hemodialysis/home, renal insufficiency, renal osteodystrophy, dialysis, kidneys/artificial, motor activity, exercise, walking, physical exertion, physical endurance, exercise tolerance, physical fitness, exercise therapy, sports, bicycling, psychomotor performance, motor skills; it also included the following keywords: renal, kidney, insufficien*, replacement*, failure*, osteodytroph*, necrosis, necroses, artificial, dialy*, microdialy*, haemodialy*, hemodialy*, intradialy*, hemodiafiltration*, haemodiafiltration*, hemo-diafiltration*, haemo-diafiltration*, cardio, muscle*, muscular, endurance, aerobic*, physical, training, capacity*, capab*, therap*, toleran*, prescri*, interven*, techni*, physical*, motion*, activit*, fit, fitness, function*, exert*, modalit*, motor, skill*, psychomotor, performance. The asterisk $(*)$ sign denotes that the word before it will be searched in all its possible versions. Boolean operators "OR, AND" were used for the search. The full search strategy is available on https://www.crd. 
york.ac.uk/PROSPEROFILES/52062_STRATEGY_20180 205.pdf.

This systematic review and meta-analysis followed the PRISMA (Preferred Reporting Items for Systematic Reviews and Meta-Analyses) guidelines [20] and was registered on Prospero (registration ID: CRD42016052062; available at: http://www.crd.york.ac.uk/PROSPERO/display_recor d.php?ID=CRD42016052062). A medical librarian validated the search strategy.

\section{Study selection}

Three reviewers (N.S., M.K., C.K.) independently assessed all retrieved articles. Overall average pairwise percent agreement between the 3 reviewers was $96.667 \%$ (Fleiss Kappa coefficient $=0.967$; Krippendorff's Alpha $=0$ ). Only one article was disagreed upon by reviewer \#3, and a consensus was reached through discussion between the 3 reviewers.

\section{Data analysis}

Three authors (N.S., M.K., C.K.) developed a template for data extraction; information extracted included patient characteristics, study design, exercise prescription and outcome variables (Kt/v, URR, serum P, QOL, vitamin D3, PTH, CRP, cost effectiveness, hospitalization rate, number of emergency HD, intake of P binders, mortality). Eligible articles were reviewed, and relevant data was then extracted, and organized into 2 main results tables by one author (N.S.).

The meta-analysis was performed using the Explanatory Software for Confidence Intervals (ESCI) program [21], based on the change in P, QOL-PCS, QOL-MCS and Kt/v which is the difference in mean between the start and end of the intervention. Pooled standard deviation was also calculated using the following formula:

$\sqrt{ }\left[(\text { SEM pre intervention })^{2}+(\text { SEM post intervention })^{2}\right]$

where $\mathrm{SEM}=\mathrm{SD} / \sqrt{ }$ sample size. Heterogeneity was assessed across studies using the diamond ratio (DR); a DR $>2$ implies considerable heterogeneity [22]. In the case of heterogeneity, a random-effects model was used. The meta-analysis was conducted for outcomes reported in a minimum of 3 studies, due to the potential for greater uncertainty with fewer studies [23]. The effect size (ES) of controlled studies was calculated, when possible, to evaluate the effectiveness of the IDE intervention. The reported ES was based on the relative size of Cohen's d, which categorizes the studies in the following four levels: $d<0.2=$ negligible effect, over $0.2=$ a small effect, over $0.5=$ a medium effect, and over $0.8=\mathrm{a}$ large effect [24]. The corresponding 95th confidence intervals (CIs) were also reported for each outcome.

\section{Results}

This systematic review was able to identify a wide range of parameters, possibly related to a positive effect of IDE, such as Kt/v, P, QOL, URR, hospitalization, and CRP. After title and abstract screening, the searches resulted in 15,092 articles, of which 52 were eligible for full text screening. The authors excluded 25 articles for one of the following reasons: no intervention was conducted, study outcomes were not measured, the study included children, had mixed types of exercise or non-aerobic exercises, had an intervention duration of $<1$ month, or the full text could not be retrieved even after contacting the authors. Thus 22 articles were included in this review (Fig. 1). Only the randomized controlled trials (RCTs) and the interventions with comparison groups with common outcomes were included in the meta-analysis (12 studies). The main characteristics of controlled and uncontrolled studies are presented respectively in Tables 2 and 3. The percentage change of all outcomes reported in Tables 2 and 3 was calculated by the authors using the pre and post outcome results using the following formula: ((Post Outcome Result - Pre Outcome Result)/Pre Outcome Result)*100.

\section{General specifications of studies}

The number of participants in a single study ranged from 6 to 194, and the mean age of participants varied between 43.3 and 72.5 years. Male gender was predominant in most studies except for $5[13,27,31,33,40,41]$. The IDE in 13 studies consisted of cycling only [1, 2, 14, 26, 28, 30, 34, 36-40, 42], and 8 studies included warm-up and stretching in addition to cycling [13, 25, 27, 29, 31-33, 35]. One study consisted of aerobic range of motion capacity only [12]. Exercise intensity was assessed in 11 studies [1, 2, 13, 25, 28, 31-34, 39, 40] using the Borg Rating of Perceived Exertion Scale, set between 10 and 16. The other studies used either the patient's maximal exercise capacity, ranging between 30 and $75 \%$ of oxygen consumption capacity $\left(\mathrm{VO}_{2} \max \right)[26,27,29,37,38]$ or the patient's individual capacity (4 studies) [12, 35-37]. One study did not report the intensity [14] and another one set the cycle speed at 60-70 revs/min [39]. Single IDE sessions varied in duration between 15 and $60 \mathrm{~min}$, a duration of $\geq 30$ min being the most used one. As for the length of the interventions, it varied between 2 and 6 months. IDE was performed at every HD session ( 2 or 3 times per week). 


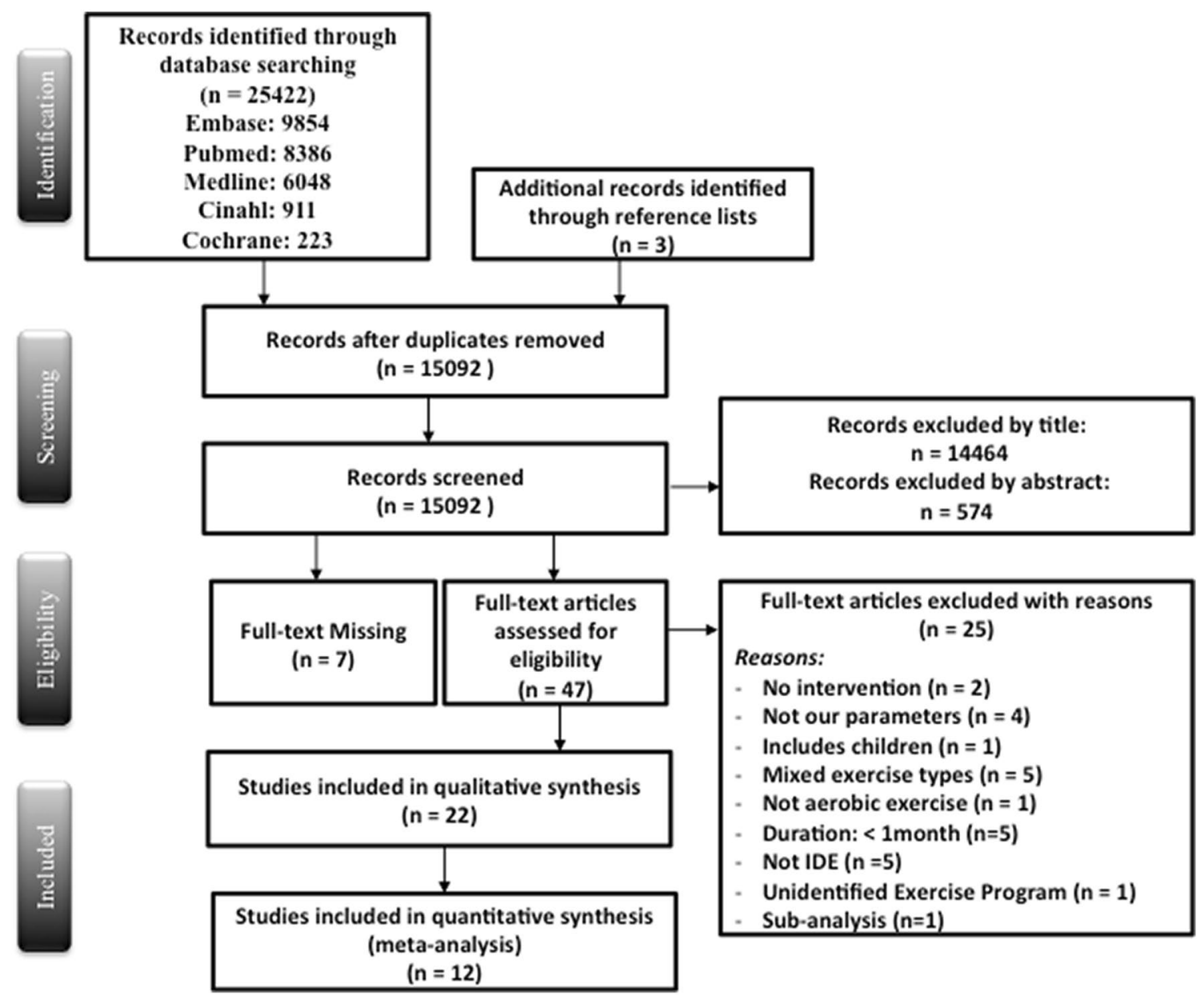

Fig. 1 Flow chart of study selection based on the PRISMA statement

\section{IDE effects on quality of life}

A total of 17 studies [1, 13, 14, 25-37, 42] showed the effect of aerobic IDE on QOL. Data were very heterogeneous due to the use of different QOL instruments. Accordingly, a meta-analysis including all studies could not be conducted. Table 2 shows detailed data from each study.

A total of 5 studies were included in the meta-analysis [26-29, 31] and reported the QOL-physical component scale (PCS) (Table 4) and the QOL-mental component scale (MCS) (Table 4), in a total number of 282 patients (214 patients in the experimental group and 68 patients in the control group). Results showed considerable heterogeneity. Overall, our meta-analysis indicated that aerobic IDE had a significant positive effect on both the QOL-PCS and QOL-MCS. In our meta-analysis, the study with large effect size for the QOL-PCS and the QOL-MCS had the youngest patients in the intervention group and the oldest in the control group [29]. The study with medium effect size for the QOL-PCS was that of Painter et al. in which the methodology used was radically different compared to the other studies, i.e. there were 2 months of conditioning exercises at home prior to the 2 months of IDE [31]. Moreover, the positive effect of IDE preconditioning on the QOL was also seen in the single arm interventional study conducted by Musavian et al. [14].

As for the total QOL score, the most significant percentage change was reported in an RCT by Wu et al. (37.7\%) where the design included $5 \mathrm{~min}$ warm-up, and $20 \mathrm{~min}$ cycling exercise for a period of 3 months at an intensity of $12-16$ points on the Borg scale [25].

Also, two single-group interventions [14, 33] had a high percentage change in the total QOL-SF-36 score: $20.8 \%$ [14] and $14.7 \%$ [33]. These latter two studies had a similar IDE program to $\mathrm{Wu}$ et al. [25] except that they had a longer duration exercise session.

\section{IDE effects on phosphorus}

Six studies [1, 12-14, 36, 40] assessed the effect of IDE on serum phosphorus (P) level. Only 3 were eligible to be included in the meta-analysis. They showed considerable 


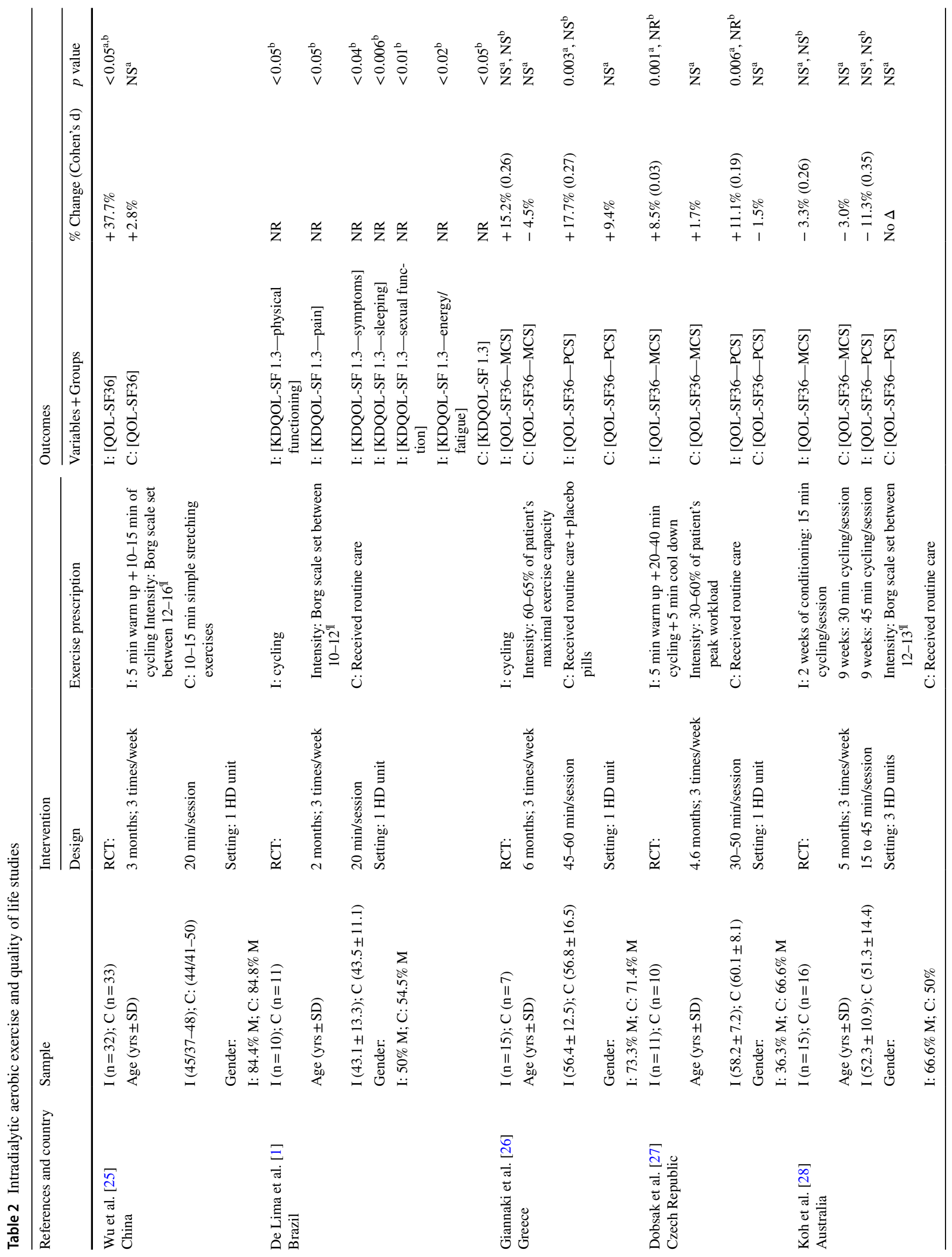




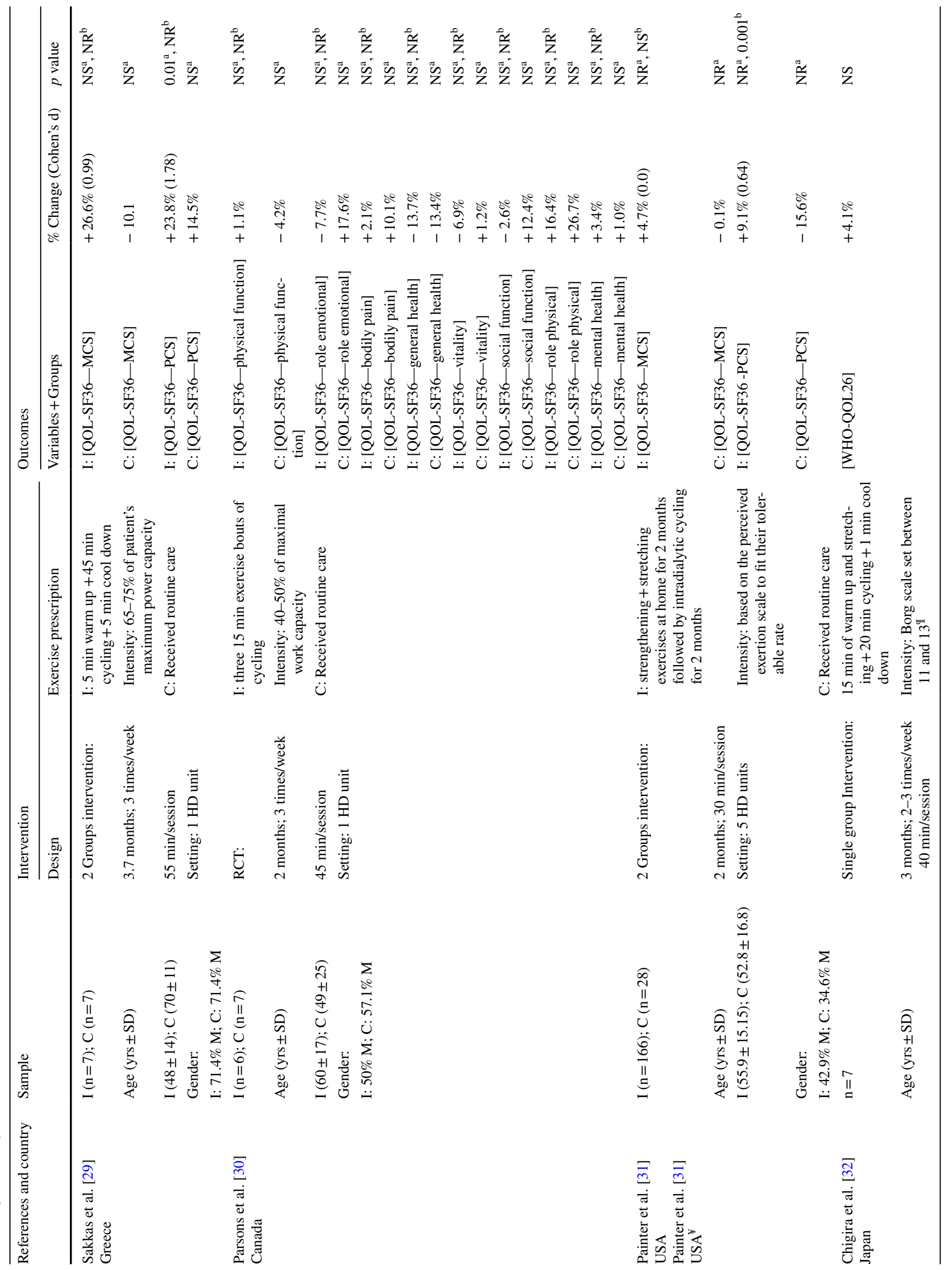




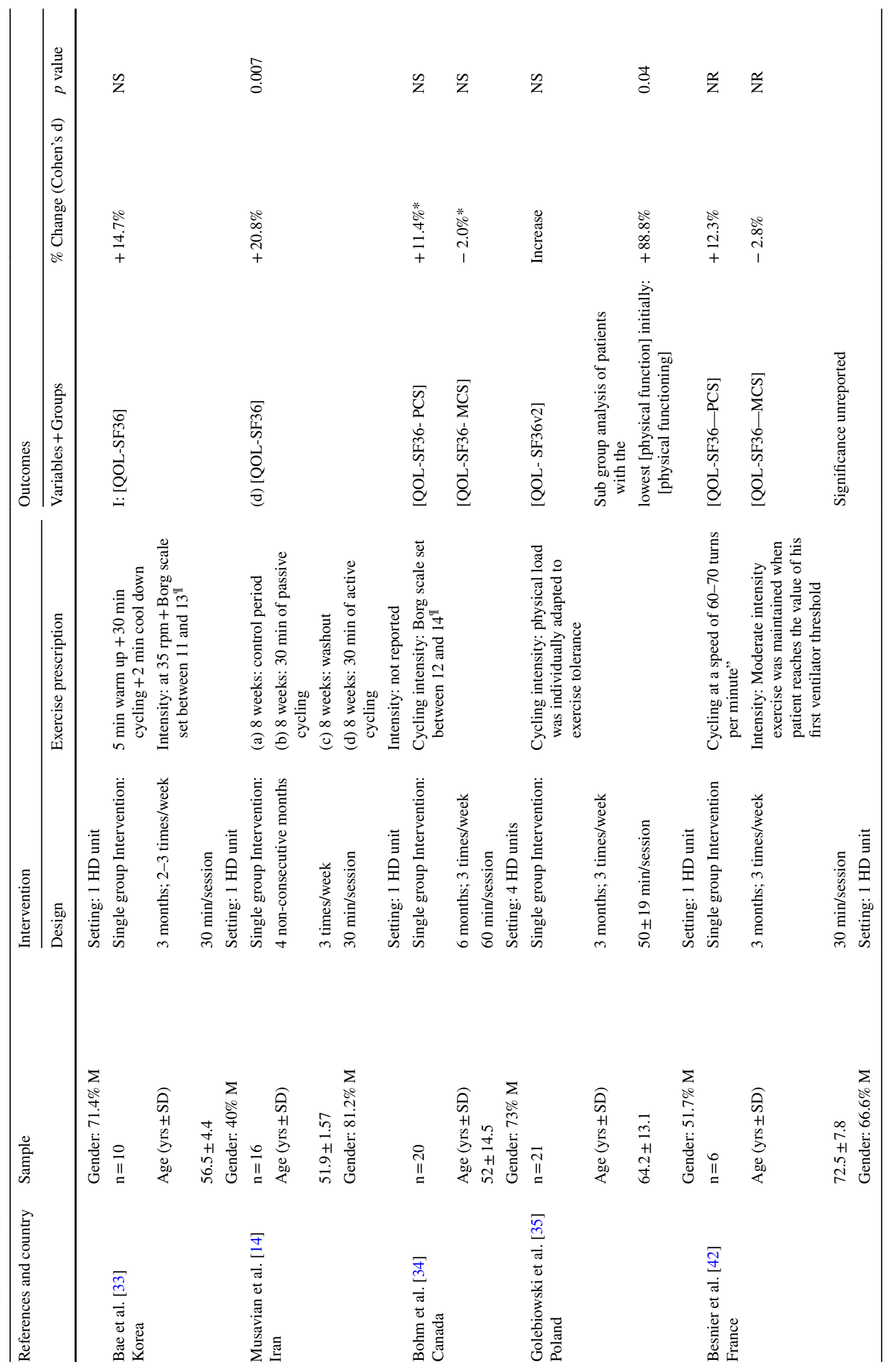




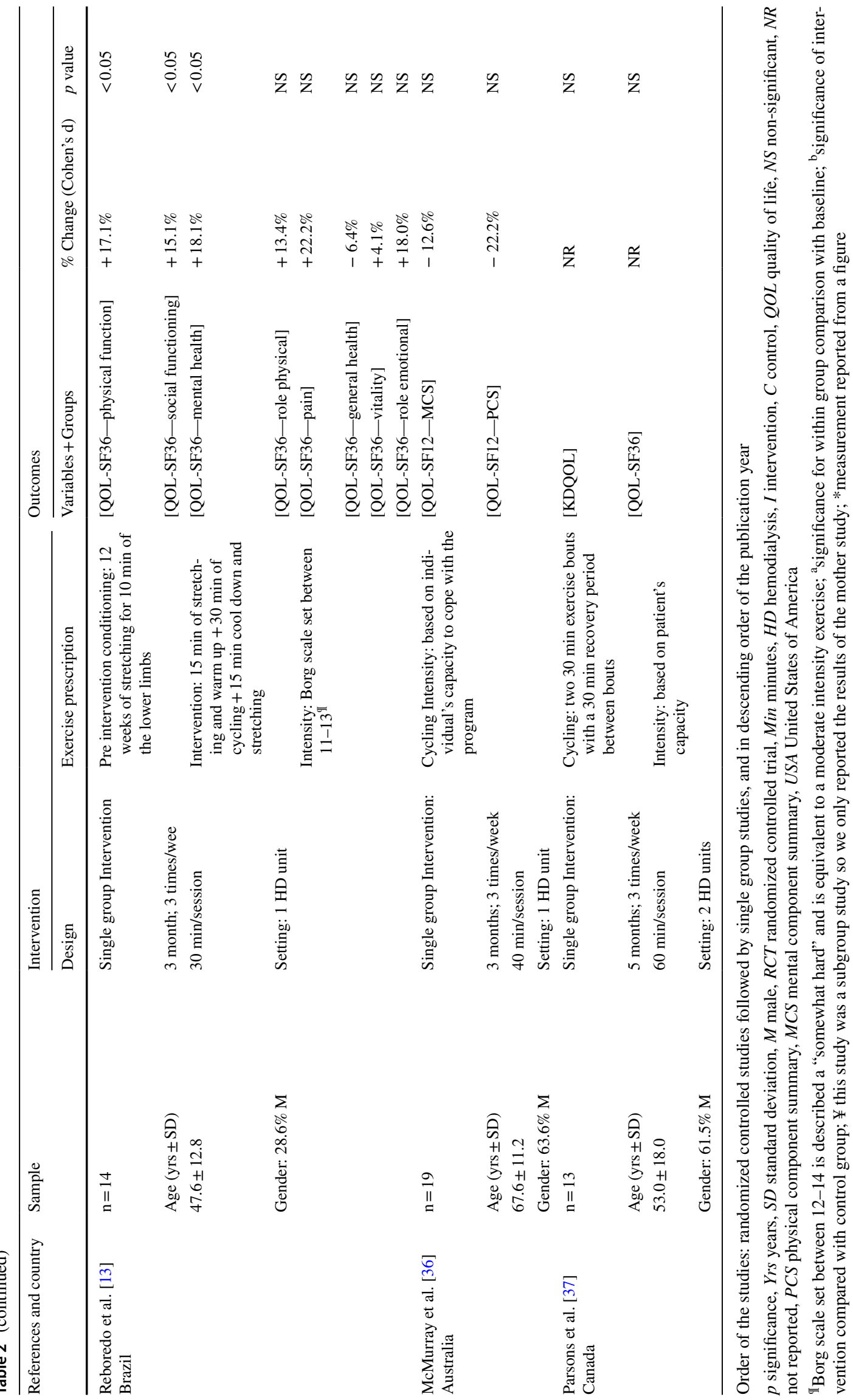


Table 3 Intradialytic aerobic exercise and biochemical/other parameters studies

\begin{tabular}{|c|c|c|c|c|c|c|}
\hline \multirow{2}{*}{$\begin{array}{l}\text { References and } \\
\text { country }\end{array}$} & \multirow[t]{2}{*}{ Sample } & \multicolumn{2}{|l|}{ Intervention } & \multicolumn{3}{|l|}{ Outcomes } \\
\hline & & Design & $\begin{array}{l}\text { Exercise prescrip- } \\
\text { tion }\end{array}$ & Variables + Groups & $\begin{array}{l}\% \text { Change (Cohen's } \\
\text { d) }\end{array}$ & $p$ value \\
\hline \multicolumn{7}{|l|}{ Dialysis efficiency } \\
\hline Groussard et al. [38] & $\mathrm{I}(\mathrm{n}=8) ; \mathrm{C}(\mathrm{n}=10)$ & RCT: & I: cycling & $\mathrm{I}:[\mathrm{Kt} / \mathrm{v}]$ & $+8.3 \%(0.95)$ & $\mathrm{NR}^{\mathrm{a}, \mathrm{b}}$ \\
\hline \multirow[t]{4}{*}{ France } & Age $(\mathrm{yrs} \pm \mathrm{SD})$ & $\begin{array}{l}3 \text { months; } 3 \text { times/ } \\
\text { week }\end{array}$ & $\begin{array}{l}\text { Intensity: } 55-60 \% \\
\text { of the peak power } \\
\text { output }\end{array}$ & $\mathrm{C}:[\mathrm{Kt} / \mathrm{v}]$ & $-10.2 \%$ & $\mathrm{NR}^{\mathrm{a}, \mathrm{b}}$ \\
\hline & $\begin{array}{l}\mathrm{I}(66.5 \pm 4.6) ; \mathrm{C} \\
\quad(68.4 \pm 3.7)\end{array}$ & $40 \mathrm{~min} / \mathrm{session}$ & $\begin{array}{l}\text { C: Received routine } \\
\text { care }\end{array}$ & & & \\
\hline & Gender: & Setting: 2 HD units & & & & \\
\hline & $\begin{array}{l}\text { I: } 62.5 \% \mathrm{M} \text {; C: } \\
77.7 \% \mathrm{M}\end{array}$ & & & & & \\
\hline Dobsak et al. [27] & $\mathrm{I}(\mathrm{n}=11) ; \mathrm{C}(\mathrm{n}=10)$ & RCT: & $\begin{array}{l}\text { I: } 5 \text { min warm up } \\
\quad+20-40 \mathrm{~min} \\
\text { cycling }+5 \mathrm{~min} \\
\text { cool down }\end{array}$ & $\mathrm{I}:[\mathrm{Kt} / \mathrm{v}]$ & $+14.6 \%(1.01)$ & $0.02^{\mathrm{a}}, \mathrm{NR}^{\mathrm{b}}$ \\
\hline \multirow[t]{4}{*}{ Czech Republic } & Age $(\mathrm{yrs} \pm \mathrm{SD})$ & $\begin{array}{l}4.6 \text { months; } 3 \text { times/ } \\
\text { week }\end{array}$ & $\begin{array}{l}\text { Intensity: } 30-60 \% \\
\text { of patient's peak } \\
\text { workload }\end{array}$ & $\mathrm{C}:[\mathrm{Kt} / \mathrm{v}]$ & $-5.6 \%$ & $\mathrm{NS}^{\mathrm{a}}, \mathrm{NR}^{\mathrm{b}}$ \\
\hline & $\begin{array}{l}\mathrm{I}(58.2 \pm 7.2) ; \mathrm{C} \\
\quad(60.1 \pm 8.1)\end{array}$ & $30-50 \mathrm{~min} / \mathrm{session}$ & $\begin{array}{l}\text { C: Received routine } \\
\text { care }\end{array}$ & I : [URR \%] & $+10.9 \%(1.44)$ & $0.001^{\mathrm{a}}, \mathrm{NR}^{\mathrm{b}}$ \\
\hline & Gender: & Setting: $1 \mathrm{HD}$ unit & & C: [URR \%] & $-3.3 \%$ & $\mathrm{NS}^{\mathrm{a}}$ \\
\hline & $\begin{array}{l}\text { I: } 36.3 \% \mathrm{M} \text {; C: } \\
\quad 66.6 \% \mathrm{M}\end{array}$ & & & & & \\
\hline Afshar et al. [39] & $\mathrm{I}(\mathrm{n}=7) ; \mathrm{C}(\mathrm{n}=7)$ & RCT: & $\begin{array}{l}\text { I: } 5 \text { min warm up } \\
\quad+10-30 \text { min } \\
\text { cycling }\end{array}$ & $\mathrm{I}:[\mathrm{Kt} / \mathrm{v}]$ & No $(0.34)$ & $\mathrm{NS}^{\mathrm{a}}, \mathrm{NR}^{\mathrm{b}}$ \\
\hline \multirow[t]{3}{*}{ Iran } & Age $(\mathrm{yrs} \pm \mathrm{SD})$ & $\begin{array}{l}2 \text { months; } 3 \text { times/ } \\
\text { week }\end{array}$ & $\begin{array}{l}\text { Intensity: Borg } \\
\text { scale set between } \\
12-16^{\mathbb{T}}\end{array}$ & $\mathrm{C}:[\mathrm{Kt} / \mathrm{v}]$ & $+0.9 \%$ & $\mathrm{NS}^{\mathrm{a}}$ \\
\hline & $\begin{array}{l}\mathrm{I}(50.7 \pm 21.06) ; \mathrm{C} \\
\quad(53 \pm 19.4)\end{array}$ & $10-30 \mathrm{~min} / \mathrm{session}$ & $\begin{array}{l}\text { C: Received routine } \\
\text { care }\end{array}$ & & & \\
\hline & Gender:100\% M & Setting: 1 HD unit & & & & \\
\hline Sakkas et al. [29] & $\mathrm{I}(\mathrm{n}=7) ; \mathrm{C}(\mathrm{n}=7)$ & $\begin{array}{l}2 \text { Groups interven- } \\
\text { tion: }\end{array}$ & $\begin{array}{l}\text { I: } 5 \text { min warm } \\
\text { up }+45 \text { min } \\
\text { cycling }+5 \text { min } \\
\text { cool down }\end{array}$ & $\mathrm{I}:[\mathrm{Kt} / \mathrm{v}]$ & $+8.3 \%(0.22)$ & $\mathrm{NS}^{\mathrm{a}}, \mathrm{NR}^{\mathrm{b}}$ \\
\hline \multirow[t]{4}{*}{ Greece } & Age $(\mathrm{yrs} \pm \mathrm{SD})$ & $\begin{array}{l}3.7 \text { months; } 3 \text { times/ } \\
\text { week }\end{array}$ & $\begin{array}{l}\text { Intensity: } 65-75 \% \\
\text { of patient's } \\
\text { maximum power } \\
\text { capacity }\end{array}$ & $\mathrm{C}:[\mathrm{Kt} / \mathrm{v}]$ & $+30 \%$ & 0.05 \\
\hline & $\begin{array}{l}\text { I }(48 \pm 14) ; C \\
\quad(70 \pm 11)\end{array}$ & $55 \mathrm{~min} / \mathrm{session}$ & $\begin{array}{l}\text { C: Received routine } \\
\text { care }\end{array}$ & & & \\
\hline & Gender: & Setting: 1 HD unit & & & & \\
\hline & $\begin{array}{l}\text { I: } 71.4 \% \mathrm{M} ; \mathrm{C}: \\
\quad 71.4 \% \mathrm{M}\end{array}$ & & & & & \\
\hline Parsons et al. [30] & $I(n=6) ; C(n=7)$ & RCT: & $\begin{array}{l}\text { I: three } 15 \mathrm{~min} \\
\text { exercise bouts of } \\
\text { cycling }\end{array}$ & $\mathrm{I}:[\mathrm{Kt} / \mathrm{v}]$ & $+0.7 \%(0.18)$ & $\mathrm{NS}^{\mathrm{a}}, \mathrm{NR}^{\mathrm{b}}$ \\
\hline \multirow[t]{3}{*}{ Canada } & Age $(\mathrm{yrs} \pm \mathrm{SD})$ & $\begin{array}{l}2 \text { months; } 3 \text { times/ } \\
\text { week }\end{array}$ & $\begin{array}{l}\text { Intensity: } 40-50 \% \\
\text { of maximal work } \\
\text { capacity }\end{array}$ & $\mathrm{C}:[\mathrm{Kt} / \mathrm{v}]$ & $-3.1 \%$ & $\mathrm{NS}^{\mathrm{a}}, \mathrm{NR}^{\mathrm{b}}$ \\
\hline & $\begin{array}{l}\text { I }(60 \pm 17) ; C \\
\quad(49 \pm 25)\end{array}$ & $45 \mathrm{~min} / \mathrm{session}$ & $\begin{array}{l}\text { C: Received routine } \\
\text { care }\end{array}$ & & & \\
\hline & Gender: & Setting: 1 HD unit & & & & \\
\hline
\end{tabular}


Table 3 (continued)

\begin{tabular}{|c|c|c|c|c|c|c|}
\hline \multirow{2}{*}{$\begin{array}{l}\text { References and } \\
\text { country }\end{array}$} & \multirow[t]{2}{*}{ Sample } & \multicolumn{2}{|l|}{ Intervention } & \multicolumn{3}{|l|}{ Outcomes } \\
\hline & & Design & $\begin{array}{l}\text { Exercise prescrip- } \\
\text { tion }\end{array}$ & Variables + Groups & $\begin{array}{l}\% \text { Change (Cohen's } \\
\text { d) }\end{array}$ & $p$ value \\
\hline & $\begin{array}{l}\text { I: } 50 \% \mathrm{M} ; \mathrm{C}: 57.1 \% \\
\mathrm{M}\end{array}$ & & & & & \\
\hline Chigira et al. [32] & $\mathrm{n}=7$ & $\begin{array}{l}\text { Single group Inter- } \\
\text { vention }\end{array}$ & $\begin{array}{l}15 \text { min of warm } \\
\text { up and stretch- } \\
\text { ing }+20 \text { min } \\
\text { cycling }+1 \text { min } \\
\text { cool down }\end{array}$ & {$[\mathrm{Kt} / \mathrm{v}]$} & $+6.6 \%$ & NS \\
\hline \multirow[t]{3}{*}{ Japan } & Age $(\mathrm{yrs} \pm \mathrm{SD})$ & $\begin{array}{l}3 \text { months; } 2-3 \\
\text { times/week }\end{array}$ & & & & \\
\hline & $70.6 \pm 4.4$ & $40 \mathrm{~min} / \mathrm{session}$ & $\begin{array}{l}\text { Intensity: Borg } \\
\text { scale set between } \\
11 \text { and } 13^{\text {I }}\end{array}$ & & & \\
\hline & Gender: $71.4 \% \mathrm{M}$ & Setting: $1 \mathrm{HD}$ unit & & & & \\
\hline Musavian et al. [14] & $\mathrm{n}=16$ & $\begin{array}{l}\text { Single group Inter- } \\
\text { vention: }\end{array}$ & $\begin{array}{l}\text { (a) } 8 \text { weeks: control } \\
\text { period }\end{array}$ & (b) $[\mathrm{Kt} / \mathrm{v}]$ & $+8.3 \%$ & NS \\
\hline \multirow[t]{4}{*}{ Iran } & $\operatorname{Age}(y r s \pm S D)$ & $\begin{array}{l}2 \text { non-consecutive } \\
\text { months }\end{array}$ & $\begin{array}{l}\text { (b) } 8 \text { weeks: } 30 \mathrm{~min} \\
\text { of passive cycling }\end{array}$ & (d) $[\mathrm{Kt} / \mathrm{v}]$ & $-4.3 \%$ & NS \\
\hline & $51.9 \pm 1.5$ & 3 times/week & $\begin{array}{l}\text { (c) } 8 \text { weeks: wash- } \\
\text { out }\end{array}$ & (b) [URR \%] & $+1.9 \%$ & NS \\
\hline & Gender: $81.2 \% \mathrm{M}$ & $30 \mathrm{~min} / \mathrm{session}$ & $\begin{array}{l}\text { (d) } 8 \text { weeks: } 30 \mathrm{~min} \\
\text { of active cycling }\end{array}$ & (d) [URR \%] & $-3.3 \%$ & NS \\
\hline & & Setting: $1 \mathrm{HD}$ unit & $\begin{array}{l}\text { Intensity: not } \\
\text { reported }\end{array}$ & & & \\
\hline Parker et al. [2] & $\mathrm{n}=102$ & $\begin{array}{l}\text { Single group Inter- } \\
\text { vention }\end{array}$ & $\begin{array}{l}\text { HD units had estab- } \\
\text { lished intradialytic } \\
\text { bicycling program } \\
\text { as part of their } \\
\text { routine care }\end{array}$ & [URR \%] & $+1.4 \%$ & 0.02 \\
\hline \multirow[t]{3}{*}{ Canada } & Age $(y r s \pm S D)$ & $\begin{array}{l}6 \text { months; } 3 \text { times/ } \\
\text { week }\end{array}$ & & & & \\
\hline & $65.6 \pm 13.5$ & $\geq 30 \mathrm{~min} / \mathrm{session}$ & $\begin{array}{l}\text { Intensity: Borg } \\
\text { scale set between } \\
12-13^{\text {II }}\end{array}$ & & & \\
\hline & Gender: $67.6 \% \mathrm{M}$ & Setting: 2 HD units & & & & \\
\hline Reboredo et al. [13] & $\mathrm{n}=14$ & $\begin{array}{l}\text { Single group Inter- } \\
\text { vention: }\end{array}$ & $\begin{array}{l}\text { Pre intervention } \\
\text { conditioning: } 12 \\
\text { weeks of stretch- } \\
\text { ing for } 10 \text { min of } \\
\text { the lower limbs }\end{array}$ & {$[\mathrm{Kt} / \mathrm{V}]$} & $+41.6 \%$ & $<0.05$ \\
\hline \multirow[t]{4}{*}{ Brazil } & $\operatorname{Age}(y r s \pm S D)$ & $\begin{array}{l}3 \text { months; } 3 \text { times/ } \\
\text { week }\end{array}$ & & & & \\
\hline & $47.6 \pm 12.8$ & $30 \mathrm{~min} / \mathrm{session}$ & $\begin{array}{l}\text { Intervention: } \\
15 \text { min of stretch- } \\
\text { ing and warm } \\
\text { up }+30 \text { min of } \\
\text { cycling }+15 \text { min } \\
\text { cool down and } \\
\text { stretching }\end{array}$ & & & \\
\hline & Gender: $28.6 \% \mathrm{M}$ & Setting: $1 \mathrm{HD}$ unit & & & & \\
\hline & & & $\begin{array}{l}\text { Intensity: Borg } \\
\text { scale set between } \\
11-13^{\mathbb{I}}\end{array}$ & & & \\
\hline
\end{tabular}


Table 3 (continued)

\begin{tabular}{|c|c|c|c|c|c|c|}
\hline \multirow{2}{*}{$\begin{array}{l}\text { References and } \\
\text { country }\end{array}$} & \multirow[t]{2}{*}{ Sample } & \multicolumn{2}{|l|}{ Intervention } & \multicolumn{3}{|l|}{ Outcomes } \\
\hline & & Design & $\begin{array}{l}\text { Exercise prescrip- } \\
\text { tion }\end{array}$ & Variables + Groups & $\begin{array}{l}\% \text { Change (Cohen's } \\
\text { d) }\end{array}$ & $p$ value \\
\hline Parsons et al. [37] & $\mathrm{n}=13$ & $\begin{array}{l}\text { Single group Inter- } \\
\text { vention: }\end{array}$ & $\begin{array}{l}\text { Cycling: two } \\
30 \text { min exercise } \\
\text { bouts with a } \\
30 \text { min recovery } \\
\text { period between } \\
\text { bouts }\end{array}$ & {$[\mathrm{Kt} / \mathrm{v}]$} & $+15.4 \%$ & $<0.05$ \\
\hline \multirow[t]{3}{*}{ Canada } & Age $(y r s \pm S D)$ & $\begin{array}{l}5 \text { months; } 3 \text { times/ } \\
\text { week }\end{array}$ & & [URR \%] & $+7.1 \%$ & $<0.05$ \\
\hline & $53.0 \pm 18.0$ & $60 \mathrm{~min} / \mathrm{session}$ & $\begin{array}{l}\text { Intensity: based on } \\
\text { patient's capacity }\end{array}$ & & & \\
\hline & Gender: $61.5 \% \mathrm{M}$ & Setting: 2 HD units & & & & \\
\hline \multicolumn{7}{|l|}{ Phosphorus } \\
\hline De Lima et al. [1] & $\mathrm{I}(\mathrm{n}=10) ; C(\mathrm{n}=11)$ & RCT: & I: cycling & I: [P mg/dl] & $-3.5 \%(0.14)$ & $\mathrm{NS}^{\mathrm{a}}$ \\
\hline \multirow[t]{4}{*}{ Brazil } & Age $(\mathrm{yrs} \pm \mathrm{SD})$ & $\begin{array}{l}2 \text { months; } 3 \text { times/ } \\
\text { week }\end{array}$ & $\begin{array}{l}\text { Intensity: Borg } \\
\text { scale set between } \\
10-12^{\mathbb{I}}\end{array}$ & $\mathrm{C}:[\mathrm{P} \mathrm{mg} / \mathrm{dl}]$ & $+3.7 \%$ & $\mathrm{NS}^{\mathrm{a}}$ \\
\hline & $\begin{array}{l}\mathrm{I}(43.1 \pm 13.3) ; \mathrm{C} \\
\quad(43.5 \pm 11.1)\end{array}$ & $20 \mathrm{~min} / \mathrm{session}$ & $\begin{array}{l}\text { C: Received routine } \\
\text { care }\end{array}$ & & & \\
\hline & Gender: & Setting: $1 \mathrm{HD}$ unit & & & & \\
\hline & $\begin{array}{l}\text { I: } 50 \% \text { M; C: } 54.5 \% \\
\text { M }\end{array}$ & & & & & \\
\hline $\begin{array}{l}\text { Makhlough et al. } \\
\text { [12] }\end{array}$ & $\mathrm{I}(\mathrm{n}=25) ; \mathrm{C}(\mathrm{n}=23)$ & RCT: & $\begin{array}{l}\text { I: Aerobic move- } \\
\text { ment exercise of } \\
\text { range capacity }\end{array}$ & I: [P mg/dl] & $-24.0 \%(0.56)$ & $0.003^{\mathrm{a}}$ \\
\hline \multirow[t]{4}{*}{ Iran } & Age $(\mathrm{yrs} \pm \mathrm{SD})$ & $\begin{array}{l}2 \text { months; } 3 \text { times/ } \\
\text { week }\end{array}$ & $\begin{array}{l}\text { Intensity: based on } \\
\text { patient's capacity }\end{array}$ & C: [P mg/dl] & $+0.5 \%$ & $\mathrm{NS}^{\mathrm{a}}, \mathrm{NR}^{\mathrm{b}}$ \\
\hline & $\begin{array}{l}\text { I }(53.3 \pm 14.27) ; C \\
\quad(56.16 \pm 10.77)\end{array}$ & $15 \mathrm{~min} / \mathrm{session}$ & $\begin{array}{l}\text { C: Received routine } \\
\text { care }\end{array}$ & & & \\
\hline & Gender: & Setting: $1 \mathrm{HD}$ unit & & & & \\
\hline & $\begin{array}{c}\text { I: } 73.9 \% \mathrm{M} \text {; C: } \\
54.2 \% \mathrm{M}\end{array}$ & & & & & \\
\hline Wilund et al. [40] & $\mathrm{I}(\mathrm{n}=8) ; \mathrm{C}(\mathrm{n}=9)$ & RCT: & I: cycling & $\mathrm{I}:[\mathrm{P} \mathrm{mg} / \mathrm{dl}]$ & $+25 \%(0.9)$ & $\mathrm{NS}^{\mathrm{a}}, \mathrm{NR}^{\mathrm{b}}$ \\
\hline \multirow[t]{4}{*}{ USA } & Age $(\mathrm{yrs} \pm \mathrm{SD})$ & $\begin{array}{l}4 \text { months; } 3 \text { times/ } \\
\text { week }\end{array}$ & $\begin{array}{l}\text { Intensity: Borg } \\
\text { scale set between } \\
12-14^{\text {I }}\end{array}$ & $\mathrm{C}:[\mathrm{P} \mathrm{mg} / \mathrm{dl}]$ & $-6.3 \%$ & $\mathrm{NS}^{\mathrm{a}}, \mathrm{NR}^{\mathrm{b}}$ \\
\hline & $\begin{array}{l}\mathrm{I}(60.8 \pm 3.2) ; \mathrm{C} \\
\quad(59.0 \pm 4.9)\end{array}$ & $45 \mathrm{~min} / \mathrm{session}$ & & & & \\
\hline & Gender: & Setting: $1 \mathrm{HD}$ unit & $\begin{array}{l}\text { C: Received routine } \\
\text { care }\end{array}$ & & & \\
\hline & $\begin{array}{c}\text { I: } 37.5 \% \mathrm{M} \text {; C: } \\
42.8 \% \mathrm{M}\end{array}$ & & & & & \\
\hline Musavian et al. [14] & $\mathrm{n}=16$ & $\begin{array}{l}\text { Single-group Inter- } \\
\text { vention: }\end{array}$ & $\begin{array}{l}\text { (a) } 8 \text { weeks: control } \\
\text { period }\end{array}$ & (b) $[\mathrm{P} \mathrm{mg} / \mathrm{dl}]$ & $-0.4 \%$ & NS \\
\hline \multirow[t]{4}{*}{ Iran } & Age $(\mathrm{yrs} \pm \mathrm{SD})$ & $\begin{array}{l}2 \text { non-consecutive } \\
\text { months }\end{array}$ & $\begin{array}{l}\text { (b) } 8 \text { weeks: } 30 \mathrm{~min} \\
\text { of passive cycling }\end{array}$ & (d) $[\mathrm{P} \mathrm{mg} / \mathrm{dl}]$ & $-13.1 \%$ & 0.004 \\
\hline & $51.9 \pm 1.57$ & 3 times/week & $\begin{array}{l}\text { (c) } 8 \text { weeks: wash- } \\
\text { out }\end{array}$ & & & \\
\hline & Gender: $81.2 \% \mathrm{M}$ & $30 \mathrm{~min} / \mathrm{session}$ & $\begin{array}{l}\text { (d) } 8 \text { weeks: } 30 \mathrm{~min} \\
\text { of active cycling }\end{array}$ & & & \\
\hline & & Setting: 1 HD unit & $\begin{array}{l}\text { Intensity: not } \\
\text { reported }\end{array}$ & & & \\
\hline
\end{tabular}


Table 3 (continued)

\begin{tabular}{|c|c|c|c|c|c|c|}
\hline \multirow{2}{*}{$\begin{array}{l}\text { References and } \\
\text { country }\end{array}$} & \multirow[t]{2}{*}{ Sample } & \multicolumn{2}{|l|}{ Intervention } & \multicolumn{3}{|l|}{ Outcomes } \\
\hline & & Design & $\begin{array}{l}\text { Exercise prescrip- } \\
\text { tion }\end{array}$ & Variables + Groups & $\begin{array}{l}\% \text { Change (Cohen's } \\
\text { d) }\end{array}$ & $p$ value \\
\hline Reboredo et al. [13] & $\mathrm{n}=14$ & $\begin{array}{l}\text { Single-group Inter- } \\
\text { vention }\end{array}$ & $\begin{array}{l}\text { Pre intervention } \\
\text { conditioning: } 12 \\
\text { weeks of stretch- } \\
\text { ing for } 10 \mathrm{~min}\end{array}$ & {$[\mathrm{P} \mathrm{mg} / \mathrm{dl}]$} & $-7.0 \%$ & NS \\
\hline \multirow[t]{4}{*}{ Brazil } & Age $(y r s \pm S D)$ & 3 months & of the lower limbs & & & \\
\hline & $47.6 \pm 12.8$ & 3 times/week & $\begin{array}{l}\text { Intervention: } \\
15 \text { min of stretch- } \\
\text { ing and warm } \\
\text { up }+30 \text { min of } \\
\text { cycling }+15 \text { min } \\
\text { cool down and } \\
\text { stretching }\end{array}$ & & & \\
\hline & Gender: $28.6 \% \mathrm{M}$ & $30 \mathrm{~min} / \mathrm{session}$ & & & & \\
\hline & & Setting: $1 \mathrm{HD}$ unit & $\begin{array}{l}\text { Intensity: Borg } \\
\text { scale set between } \\
11-13^{\text {I }}\end{array}$ & & & \\
\hline $\begin{array}{l}\text { McMurray et al. } \\
\text { [36] }\end{array}$ & $\mathrm{n}=19$ & $\begin{array}{l}\text { Single-group Inter- } \\
\text { vention: }\end{array}$ & Cycling & [P mmol/l] & $-2.7 \%$ & NS \\
\hline \multirow[t]{3}{*}{ Australia } & Age $(\mathrm{yrs} \pm \mathrm{SD})$ & $\begin{array}{l}3 \text { months; } 3 \text { times/ } \\
\text { week }\end{array}$ & $\begin{array}{l}\text { Intensity: based } \\
\text { on individual's } \\
\text { capacity to cope } \\
\text { with the program }\end{array}$ & & & \\
\hline & $67.6 \pm 11.2$ & $40 \mathrm{~min} / \mathrm{session}$ & & & & \\
\hline & Gender: $63.6 \% \mathrm{M}$ & Setting: $1 \mathrm{HD}$ unit & & & & \\
\hline \multicolumn{7}{|l|}{ Others } \\
\hline Afshar et al. [39] & $\mathrm{I}(\mathrm{n}=7) ; \mathrm{C}(\mathrm{n}=7)$ & RCT: & $\begin{array}{l}\text { I: } 5 \text { min warm up } \\
\quad+10-30 \text { min } \\
\text { cycling }\end{array}$ & I: [hs-CRP mg/l] & $-83.8 \%$ & $\mathrm{Sig}^{\mathrm{a}}, 0.005^{\mathrm{b}}$ \\
\hline \multirow[t]{3}{*}{ Iran } & Age $(y r s \pm S D)$ & $\begin{array}{l}2 \text { months; } 3 \text { times/ } \\
\text { week }\end{array}$ & $\begin{array}{l}\text { Intensity: Borg } \\
\text { scale set between } \\
12-16^{\text {I }}\end{array}$ & C: [hs-CRP mg/l] & $+1.4 \%$ & $\mathrm{NS}^{\mathrm{a}}$ \\
\hline & $\begin{array}{l}\text { I }(50.7 \pm 21.06) ; C \\
\quad(53 \pm 19.4)\end{array}$ & $10-30 \mathrm{~min} / \mathrm{session}$ & $\begin{array}{l}\text { C: Received routine } \\
\text { care }\end{array}$ & & & \\
\hline & Gender: $100 \% \mathrm{M}$ & Setting: $1 \mathrm{HD}$ unit & & & & \\
\hline Wilund et al. [40] & $\mathrm{I}(\mathrm{n}=8) ; \mathrm{C}(\mathrm{n}=9)$ & RCT: & I: cycling & I: CRP (mg/dl) & $-5.7 \%$ & $\mathrm{NS}^{\mathrm{a}}, \mathrm{NR}^{\mathrm{b}}$ \\
\hline \multirow[t]{4}{*}{ USA } & Age $(\mathrm{yrs} \pm \mathrm{SD})$ & $\begin{array}{l}4 \text { months; } 3 \text { times/ } \\
\text { week }\end{array}$ & $\begin{array}{l}\text { Intensity: Borg } \\
\text { scale set between } \\
12-14^{\text {I }}\end{array}$ & C: CRP (mg/dl) & $-3.2 \%$ & $\mathrm{NS}^{\mathrm{a}}, \mathrm{NR}^{\mathrm{b}}$ \\
\hline & $\begin{array}{l}\mathrm{I}(60.8 \pm 3.2) ; \mathrm{C} \\
\quad(59.0 \pm 4.9)\end{array}$ & $45 \mathrm{~min} / \mathrm{session}$ & & & & \\
\hline & Gender: & Setting: $1 \mathrm{HD}$ unit & $\begin{array}{l}\text { C: Received routine } \\
\text { care }\end{array}$ & & & \\
\hline & $\begin{array}{c}\text { I: } 37.5 \% \mathrm{M} \text {; C: } \\
42.8 \% \mathrm{M}\end{array}$ & & & & & \\
\hline $\begin{array}{l}\text { Golebiowski et al. } \\
\text { [35] }\end{array}$ & $\mathrm{n}=21$ & $\begin{array}{l}\text { Single-group Inter- } \\
\text { vention: }\end{array}$ & Cycling & [CRP mg/l] & $+15.0 \%$ & NS \\
\hline \multirow[t]{3}{*}{ Poland } & $\operatorname{Age}(\mathrm{yrs} \pm \mathrm{SD})$ & $\begin{array}{l}3 \text { months; } 3 \text { times/ } \\
\text { week }\end{array}$ & $\begin{array}{l}\text { Intensity: physical } \\
\text { load was individu- } \\
\text { ally adapted to } \\
\text { exercise tolerance }\end{array}$ & & & \\
\hline & $64.2 \pm 13.1$ & $50 \pm 19 \mathrm{~min} / \mathrm{session}$ & & & & \\
\hline & Gender: $51.7 \% \mathrm{M}$ & Setting: 1 HD unit & & & & \\
\hline
\end{tabular}


Table 3 (continued)

\begin{tabular}{|c|c|c|c|c|c|c|}
\hline \multirow{2}{*}{$\begin{array}{l}\text { References and } \\
\text { country }\end{array}$} & \multirow[t]{2}{*}{ Sample } & \multicolumn{2}{|l|}{ Intervention } & \multicolumn{3}{|l|}{ Outcomes } \\
\hline & & Design & $\begin{array}{l}\text { Exercise prescrip- } \\
\text { tion }\end{array}$ & Variables + Groups & $\begin{array}{l}\% \text { Change (Cohen's } \\
\text { d) }\end{array}$ & $p$ value \\
\hline Parker et al. [2] & $\mathrm{n}=102$ & $\begin{array}{l}\text { Single-group Inter- } \\
\text { vention }\end{array}$ & $\begin{array}{l}\text { HD units had estab- } \\
\text { lished intradialytic } \\
\text { bicycling program } \\
\text { as part of their } \\
\text { routine care }\end{array}$ & $\begin{array}{l}\text { [Hospitalization } \\
\text { rate] }\end{array}$ & $-2.6 \%$ & NS \\
\hline \multirow[t]{3}{*}{ Canada } & $\operatorname{Age}(\mathrm{yrs} \pm \mathrm{SD})$ & $\begin{array}{l}\text { (Retrospective } \\
\text { Study) }\end{array}$ & & & & \\
\hline & $65.6 \pm 13.5$ & $\begin{array}{l}6 \text { months; } 3 \text { times/ } \\
\text { week }\end{array}$ & $\begin{array}{l}\text { Intensity: Borg } \\
\text { scale set between } \\
12-13^{\text {II }}\end{array}$ & & & \\
\hline & Gender: $67.6 \% \mathrm{M}$ & $\begin{array}{l}\geq 30 \mathrm{~min} / \mathrm{session} \\
\text { Setting: } 2 \text { HD units }\end{array}$ & & & & \\
\hline
\end{tabular}

Order of the studies: randomized controlled studies followed by single group studies, and in descending order of the publication year

$p$ significance, $Y r s$ years, $S D$ standard deviation, $M$ male, $R C T$ randomized controlled trial, Min minutes, $H D$ hemodialysis, $I$ intervention, $C$ control, Sig significant, $N S$ non-significant, $N R$ not reported, $P$ phosphorus, $U R R$ urea reduction ration, $P R U$ percentage reduction ration, $C R P$ C-reactive protein

${ }^{\mathrm{a}}$ significance for within group comparison with baseline; ${ }^{\mathrm{b}}$ significance of intervention compared with control group; ${ }^{\text {IB }} \mathrm{Borg}$ scale set between $12-14$ is described a "somewhat hard" and is equivalent to a moderate intensity exercise

heterogeneity, and indicated that IDE had no significant effect on serum P levels (Table 4). Makhlough et al. reported the highest positive effect size and was the only to show a significant $\mathrm{P}$ decrease [12]; their intervention consisted of aerobic range of motion capacity for $15 \mathrm{~min} / \mathrm{session}$ for 2 months. This study was also unique in the fact of having the highest mean baseline serum P $(7.68 \mathrm{mg} / \mathrm{dl})$ for the IDE group compared to the other studies included in this systematic review. Contrary to the other 2 RCTs [1, 12], Wilund et al. showed an increase in the P level post IDE; the study had the most prolonged intervention period (4 months) and exercise duration ( $45 \mathrm{~min}$ ), yet the lowest sample size; the mean starting value for serum $\mathrm{P}$ in the intervention group was $5.2 \mathrm{mg} / \mathrm{dl}$ [40].

As for the other 3 single-group interventions [13, 14, 36], serum $P$ levels were lower at the end of the study, the decrease being statistically significant only in the shortest duration intervention [14], where patients exercised for $30 \mathrm{~min} / \mathrm{session}$ (but the intensity was not reported). It is worth noting that the 3 studies all started with a P level ranging between 4.82 and $5.7 \mathrm{mg} / \mathrm{dl}$.

\section{IDE effects on dialysis efficiency}

Ten studies assessed the effect of IDE on Kt/V [13, 14, 27, 29, 30, 32, 37-39] and URR [2, 14, 27, 37]. Only 5 were eligible for the meta-analysis [27, 29-31, 38], and they showed considerable heterogeneity. Their pooled analysis showed that IDE had no significant effect on Kt/V (Table 4). Nevertheless, Dobsak et al. was able to show a large effect size and a positive change $(+14.6 \%)$ on $\mathrm{Kt} / \mathrm{V}$ [27]. This study was unique in its gender distribution ( $63.7 \%$ of the intervention group were females), duration (4.6 months), intensity (30-60\%), and session length (30-50 min). Another study conducted by Groussard et al. had a large effect size with a positive change $(+8.3 \%)$ on Kt/v [38]. Two other studies also reported a positive change in $\mathrm{Kt} / \mathrm{v}$ post intervention but had a small effect size [29, 30].

Among the single-group interventions, the 2 studies that showed the most significant change in $\mathrm{Kt} / \mathrm{v}$ were those by Reboredo et al. and Parsons et al. [13, 37]; the former study included 3 months IDE preconditioning before the intervention and the latter one had the longest IDE duration per session and intervention length. Concerning URR, one RCT showed a large effect size and reported a positive change $(+10.9 \%)$ in URR post intervention [27]; the single-group intervention study of Parsons et al. also showed a positive URR change and had the longest session length $(60 \mathrm{~min})$ [37].

\section{Effect of IDE on inflammatory status variables}

Three studies assessed the effect of IDE on CRP [35, 39, 40]. Only 2 were RCTs [39, 40] and showed a decrease in CRP level contrary to the single-group pilot study [35] where CRP increased but not significantly. Afshar et al.'s study showed the most significant change and was unique in its gender distribution, being $100 \%$ male [39]. A meta-analysis was not possible due to the low number of eligible studies reporting on CRP. 
Table 4 Forest plot of a random effect meta-analysis assessing the change in QOL-PCS, QOL-MSC, Kt/v and P in IDE interventions versus controls

\begin{tabular}{|c|c|c|c|c|c|c|c|c|c|c|}
\hline & \multicolumn{3}{|c|}{ IDE } & \multicolumn{3}{|c|}{ No IDE } & & & & Forest Plot \\
\hline $\begin{array}{l}\text { Study } \\
\text { (duration) }\end{array}$ & $\mathbf{N}$ & $\begin{array}{l}\text { Mean } \\
\text { diff. }\end{array}$ & SD* & $\mathbf{N}$ & $\begin{array}{l}\text { Mean } \\
\text { diff. }\end{array}$ & SD* & $\begin{array}{l}\text { Mean Diff (CI) } \\
\text { Random Effect }\end{array}$ & $\begin{array}{l}\text { Study } \\
\text { Wt }\end{array}$ & $\begin{array}{l}\text { Effect } \\
\text { Size }\end{array}$ & \multirow{5}{*}{\begin{tabular}{cc|ccc}
-4 & -2 & 0 & 2 & 4 \\
- & -2 & &
\end{tabular}} \\
\hline \multicolumn{10}{|c|}{ Phosphorus } & \\
\hline $\begin{array}{l}\text { Wilund et al. } \\
(2010) \\
(4 \text { months })\end{array}$ & 8 & 1.3 & 0.3 & 9 & -0.4 & 0.3 & $1.7(1.38-2.01)$ & 36.1 & Large & \\
\hline $\begin{array}{l}\text { Makhlough et al. } \\
(2012) \\
(2 \text { months })\end{array}$ & 25 & 5.83 & 2.37 & 23 & 7.08 & 2.07 & $-1.25(-2.54-0.04)$ & 31.5 & Medium & \\
\hline $\begin{array}{l}\text { De Lima et al. } \\
(2013) \\
(2 \text { months }) \\
\end{array}$ & 10 & 5.4 & 1.7 & 11 & 5.6 & 0.9 & $-0.2(-1.42-1.02)$ & 32.3 & Small & \\
\hline \multicolumn{10}{|c|}{ TOTAL: 3 studies; N (IDE) = 43; N (No IDE) = 43; Total Mean Diff (CI): $1.9(-1.78-2.09) ; p=0.87$} & Favor IDE Favor No IDE \\
\hline \multicolumn{10}{|c|}{ QOL - PCS } & \\
\hline $\begin{array}{l}\text { Sakkas et al. } \\
(2008) \\
(3.7 \text { months) }\end{array}$ & 7 & 16 & 6.95 & 7 & 7 & $\begin{array}{l}11.3 \\
4\end{array}$ & $9(-1.953-19.953)$ & 11.3 & Large & \multirow{6}{*}{ 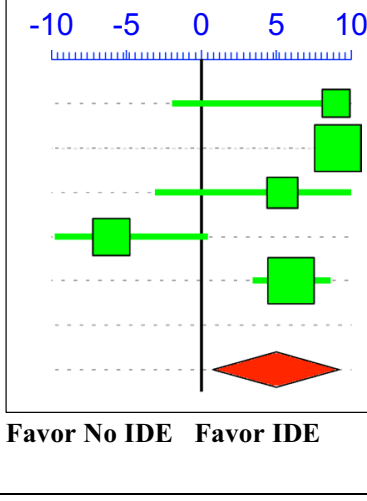 } \\
\hline $\begin{array}{l}\text { Painter et al. } \\
(2000) \\
(2 \text { months })\end{array}$ & 166 & 3.2 & 1.2 & 28 & -5.9 & 2.5 & $9.1(8.51-9.68)$ & 29.4 & Medium & \\
\hline $\begin{array}{l}\text { Giannaki et al. } \\
\text { (2013) } \\
\text { (6 months) }\end{array}$ & 15 & 11.5 & 6.3 & 7 & 6.1 & 13.1 & $5.4(-3.10-13.9)$ & 14.3 & Small & \\
\hline $\begin{array}{l}\text { Koh et al. } \\
(2010) \\
(5 \text { months }) \\
\end{array}$ & 15 & -6 & 7.7 & 16 & 0 & 9.6 & $-6.0(-12.42-0.42)$ & 18.1 & Small & \\
\hline $\begin{array}{l}\text { Dobsak et al. } \\
\text { (2012) } \\
(4.6 \text { months) }\end{array}$ & 11 & 5.2 & 2.1 & 10 & -0.8 & 3.5 & $6(3.39-8.60)$ & 26.9 & $\begin{array}{l}\text { Negli- } \\
\text { gible }\end{array}$ & \\
\hline \multicolumn{10}{|c|}{ TOTAL: 5 studies; N (IDE) = 214; N (No IDE) = 68; Total Mean Diff (CI): $4.21(0.77-9.20) ; p=0.02$} & \\
\hline \multicolumn{10}{|c|}{ QOL - MCS } & \multirow{7}{*}{ 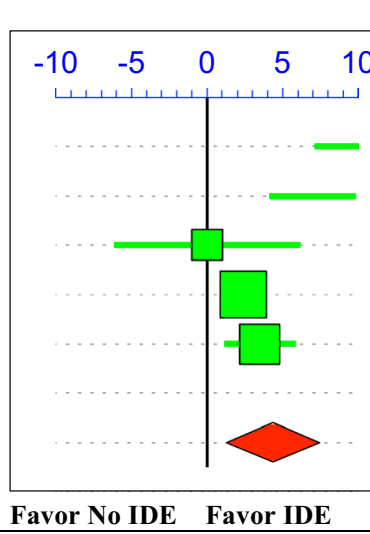 } \\
\hline $\begin{array}{l}\text { Sakkas et al. } \\
\text { (2008) } \\
\text { (3.7 months) }\end{array}$ & 7 & 16 & 13 & 7 & -6 & 12.6 & $3.07(1.29-7.45)$ & 4.6 & Large & \\
\hline $\begin{array}{l}\text { Giannaki et al. } \\
\text { (2013) } \\
\text { (6 months) } \\
\end{array}$ & 15 & 9.3 & 7.5 & 7 & 3.1 & 11 & $12.4(4.09-20.70)$ & 11.0 & Small & \\
\hline $\begin{array}{l}\text { Koh et al. } \\
(2010) \\
(5 \text { months }) \\
\end{array}$ & 15 & -2 & 7.7 & 16 & -2 & 9 & $0(-6.17-6.17)$ & 15.8 & Small & \\
\hline $\begin{array}{l}\text { Painter et al. } \\
(2000) \\
(2 \text { months })\end{array}$ & 166 & 2.3 & 1.2 & 28 & -0.1 & 2.6 & $2.4(1.80-2.99)$ & 37.1 & $\begin{array}{l}\text { Negli- } \\
\text { gible }\end{array}$ & \\
\hline $\begin{array}{l}\text { Dobsak et al. } \\
(2012) \\
(4.6 \text { months })\end{array}$ & 11 & 4.5 & 1.9 & 10 & 1 & 3.2 & $3.5(1.12-5.87)$ & 31.5 & $\begin{array}{l}\text { Negli- } \\
\text { gible }\end{array}$ & \\
\hline \multicolumn{10}{|c|}{ TOTAL: 5 studies; $N($ IDE $)=214 ;$ N $($ No IDE $)=68 ;$ Total Mean Diff $(C I): 3.07(1.29-7.45) ; p=0.05$} & \\
\hline \multicolumn{10}{|c|}{$K t / \mathbf{v}$} & \multirow{6}{*}{$\begin{array}{rrr}-0.5 & 0 & 0.5 \\
\longleftarrow,+,+, \ldots,+\end{array}$} \\
\hline $\begin{array}{l}\text { Dobsak et al. } \\
(2012) \\
(4.6 \text { months }) \\
\end{array}$ & 11 & 0.21 & 0.1 & 10 & -0.08 & 0.2 & $0.29(0.14-0.43)$ & 19.8 & Large & \\
\hline $\begin{array}{l}\text { Groussard et al. } \\
(2015) \\
(3 \text { months })\end{array}$ & 8 & 0.11 & 0.05 & 10 & 0.15 & 0.04 & $0.26(0.21-0.30)$ & 21.4 & Large & \\
\hline $\begin{array}{l}\text { Parsons et al. } \\
(2004) \\
(2 \text { months }) \\
\end{array}$ & 6 & 0.01 & 0.15 & 7 & -0.04 & 0.12 & $0.05(-0.11-0.21)$ & 19 & Small & \\
\hline $\begin{array}{l}\text { Afshar et al. } \\
(2010) \\
(2 \text { months })\end{array}$ & 7 & 0 & 0.17 & 7 & 0.01 & 0.14 & $-0.01(-0.19-0.17)$ & 19.4 & Small & \\
\hline $\begin{array}{l}\text { Sakkas et al. } \\
(2008) \\
(3.7 \text { months })\end{array}$ & 7 & 0.1 & 0.1 & 7 & 0.3 & 0.1 & $-0.2(-0.31--0.08)$ & 20.4 & Small & \\
\hline TOTAL: 5 stt & $\mathbf{s} ; \mathbf{N}$ & E) $=39$ & (No & $\mathbf{D}$ & 41; 1 & & & & & Favor No IDE Favor IDE \\
\hline
\end{tabular}
Abbreviations: CI: confidence interval; diff.: difference; * pooled SD 


\section{Effect of IDE on hospitalizations}

Hospitalization rate was reported in only 1 single-group intervention [2], and showed a non-significant decrease over 6 months.

\section{Effect of IDE on other outcomes}

None of the articles identified through our screening methodology measured vitamin D3, PTH, cost effectiveness, number of emergency HD, intake of P binders, and mortality, which were initially planned to be assessed in this systematic review. Thus, we cannot report on these parameters.

\section{Discussion}

This systematic review focused on the effect of aerobic IDE programs in an HD setting. The conclusions drawn from this review are not only based on the meta-analysis results, since some of our main outcome parameters were trialed only in studies without comparators.

A meta-analysis of 5 articles showed that aerobic IDE improves both QOL-PCS and QOL-MCS. This is partly in line with findings reported in the literature, where the metaanalysis of studies on aerobic and resistance IDE programs showed that IDE had a significant difference on the QOLPCS but not on the QOL-MCS $[8,10]$. Pre-IDE muscle conditioning and young age were identified as the key components of success in terms of patients being able to maintain a long-term IDE program and thus harvest the positive clinical outcomes [14, 31]. However, a recent review conducted by Gomes et al. showed that aerobic IDE was not associated with improvement in the QOL [43]. However, Gomes et al.'s meta-analysis included an article in which IDE targeted malnourished patients; thus it has different selection criteria and its findings cannot be generalized to the general HD population. Our review, however, included 1 article with a large sample size that might have affected the results. In addition, our meta-analysis was based on the outcome change rather than on post intervention values.

In our review we could not identify an overall effect of IDE on P levels in HD patients. However, a possible effective IDE recipe to improve serum $P$ was identified by Makhlough et al. [12] where patients included in the study were hyperphosphatemic (serum $\mathrm{P}>4.5 \mathrm{mg} / \mathrm{dl}$ ) [44] and the exercise involved was a 2-month aerobic exercise program involving range of motion capacity. Moreover, Musavian et al. had an added value of preconditioning exercises before implementation of IDE programs on their serum $\mathrm{P}$ results [14]. These results may be explained by the fact that hyperphosphatemic patients are the ones that need additional therapies to improve their current status, thus they would be the ones that reap the effect of IDE the most [14, 44]. Out of the 3 RCTs focusing on serum P, two of them had an increase in $\mathrm{P}$ in their control groups, thus we postulate that the IDE program reversed the natural deterioration of serum $\mathrm{P}$ commonly seen among HD patients; this gives more importance to the positive clinical effect of IDE. HD patients have numerous comorbidities reducing the magnitude of improvement [31]; this very fact may be more obvious when the parameter taken into account is initially in the normal range. Wilund et al. had the highest exercise duration and length of intervention, but the $\mathrm{P}$ level increased at the end of the intervention [40]. The authors did not define the mechanisms of this increase and mentioned their small sample size as a limitation, preventing control for various factors. In addition, Wilund et al. did not report if dietary $\mathrm{P}$ was monitored.

Our review did not find an overall positive effect of IDE on $\mathrm{Kt} / \mathrm{v}$ in HD patients. On the other hand, time seems to be the key for Kt/V improvement with IDE. When comparing the 5 RCTs, characterized by similar duration of exercise per dialysis session and intensity, the most prolonged exercise program [27] resulted in the most significant change. Also, gender may play a role since the only intervention that showed no change was in a study on male patients only. Among the single-group interventions, Reboredo et al. showed the largest positive significant change $(+41.6 \%)$ in $\mathrm{Kt} / \mathrm{V}$ [13]; this study had the youngest patients, and the highest female gender percentage among the other comparable studies [14, 32, 37]. It included conditioning before intervention and stretching along with cycling. Another meta-analysis [8] showed that IDE groups had higher $\mathrm{Kt} / \mathrm{v}$ values than the control group; however, the type of exercise included also resistance and stretching [31, 45, 46], not only aerobic exercise. Unlike Sheng et al., we were not able to identify a clear effect of aerobic IDE on Kt/V. Thus we cannot exclude that other exercise modalities could be more beneficial.

There was no association between IDE and mortality risk or rates of hospitalization in the included studies; this was also reported in a previous systematic review [11] where no RCT on exercise training with chronic kidney disease patients reported exercise as a direct cause of death. Nephrologists should capitalize on this fact to advocate exercise to their patients. Nevertheless, data insufficiency in this field may be a major source of hesitation in advocating IDE.

Other factors beyond those studied in the present paper may provide arguments in favor of IDE. After the initiation of HD therapy, patients' lean tissue mass tends to decrease and fat tissue mass and body mass index (BMI) tend to increase [47], which may contribute to sarcopenic obesity [48]. Fluctuation of fat and lean tissue is affected by gender, comorbidities and the initial body composition [47]. Thus, assessing the body composition in HD patients is crucial, especially since BMI is not always a good 
Table 5 Putative recipe for aerobic IDE

$\begin{array}{ll}\text { Preconditioning } & \begin{array}{l}\text { Strengthening and stretching exercise } 2 \text { months } \\ \text { prior to exercise initiation }\end{array} \\ \text { Modality } & \text { Warm up: Range capacity exercise + Cycling } \\ \text { Frequency } & 3 \text { times per week } \\ \text { Intensity } & \text { Borg scale average rating of } 12 \text { points } \\ & \text { Or } 60-65 \% \text { of the peak power output } \\ \text { Duration } & \begin{array}{l}\text { Range capacity exercises: } 15 \text { min }+ \text { Cycling: } 30-45 \\ \text { min }\end{array}\end{array}$

indicator in this population [49]. Mortality is higher in HD patients with a low fat tissue index (FTI) and lean tissue index (LTI) [49]; whereas survival is higher in patients with fat and lean tissue compartments lying between the 10th and 90th percentile of a healthy population [49]. It has also been shown that physical inactivity is, in combination with a decline in lean tissue, related to muscle weakness [50] which is an important determinant of outcome in dialysis patients [51]. Whereas resistance-based IDE was shown to increase muscle strength and quadriceps cross-sectional area, its effect on LTI was less apparent, this in contrast to treatment with anabolic steroids [52]. Therefore, assessing body composition as well as muscle strength on a routine basis may be useful for both risk stratification as well as targeted interventions.

Cardiovascular diseases are a major comorbidity in HD patients [53]. National and international guidelines for cardiac rehabilitation include education, exercise training and psychological support [54] as part of their programs. HD patients are mostly cardiac patients; therefore, like the cardiologist, nephrologists should be encouraged to focus on their patient's behavioral goals through counseling and follow-ups.

HD patients spend an average of $12 \mathrm{~h}$ weekly being sedentary on dialysis; thus it is a good opportunity to integrate IDE. IDE might not be a miraculous cure, but it could surely add more functionality to the time spent in HD, improve patients' QOL, decrease anxiety, and maybe increase adherence to the treatment. Perhaps, IDE could be coupled with music, since music therapy in HD patients has shown it can reduce anxiety [55], pain and nausea [56], while it improves blood pressure, quality of sleep, fever, cramps, anxiety and depression levels, pain, and itching [57].

Based on the studies that showed statistically significant positive effects of IDE on all study parameters in our review, a putative recipe for aerobic exercise in chronic hemodialysis patients could be suggested (Table 5). Exercise, specifically intradialytic, should be part of the HD treatment protocol. Guidelines are required for IDE to be an adjunct therapy to HD.

\section{Limitations}

The limitations of this review were the lack of quality analysis of the studies. As for the general limitations related to the available published literature, these ranged from the selection of the healthier HD patients, to the scarcity of the available interventions, to the limited number of eligible interventions for meta-analysis, to the diversity in exercise intensity, duration and modality across the studies. Furthermore, the presence of a publication bias in this new researched field cannot be underestimated. The authors could not report on all the primary and secondary outcomes that they had planned to evaluate because some of these were unavailable in the selected studies.

\section{Conclusions}

In conclusion, the results of this systematic review suggest that aerobic IDE did not impose any health hazard in HD patients and its incorporation into clinical practice can result in significant improvement in QOL-PCS and QOL-MCS [26-29, 31]. Since sarcopenic obesity is a prevalent phenomenon among HD patients, this can be a call for making IDE a routine practice in HD units. But the accurate mode of delivery (intensity, type, methods) needs to be tailored to each subgroup of patients within the context of the country, culture, and healthcare system.

Future research should assess the long-term effectiveness and safety of IDE. Most of the studies were conducted in Western countries and thus more insights are needed concerning other regions, such as the Middle East. Last but not least, the mortality rate related to IDE should be investigated in order to strengthen the rationale for IDE programs.

Acknowledgements There was no external funding provided for this study, and the authors have no financial interests to declare. The authors acknowledge the collaboration of researchers in providing us with data on their trials that was missing in their publications. The authors would like to thank the medical information specialist, Mrs. Aida Farha, for assisting in the retrieval of the articles from the selected databases.

\section{Compliance with ethical standards}

Conflict of interest On behalf of all authors, the corresponding author states that there is no conflict of interest.

Ethical approval For this type of study, ethical approval is not required. Informed consent For this type of study, formal consent is not required.

OpenAccess This article is distributed under the terms of the Creative Commons Attribution 4.0 International License (http://creativeco mmons.org/licenses/by/4.0/), which permits unrestricted use, distribution, and reproduction in any medium, provided you give appropriate credit to the original author(s) and the source, provide a link to the Creative Commons license, and indicate if changes were made. 


\section{References}

1. de Lima MC, Cicotoste Cde L, Cardoso Kda S, Forgiarini LA, Jr Monteiro MB, Dias AS (2013) Effect of exercise performed during hemodialysis: strength versus aerobic. Ren Fail 35(5):697704. https://doi.org/10.3109/0886022X.2013.780977

2. Parker K, Zhang X, Lewin A, MacRae JM (2015) The association between intradialytic exercise and hospital usage among hemodialysis patients. Appl Physiol Nutr Metab 40(4):371-378. https://doi.org/10.1139/apnm-2014-0326

3. Levin A, Stevens PE (2014) Summary of KDIGO 2012 CKD Guideline: behind the scenes, need for guidance, and a framework for moving forward. Kidney Int 85(1):49-61. https://doi. org/10.1038/ki.2013.444

4. Young HM, Hudson N, Clarke AL, Dungey M, Feehally J, Burton JO, Smith AC (2015) Patient and staff perceptions of intradialytic exercise before and after implementation: a qualitative study. PLoS One 10(6):e0128995. https://doi.org/10.1371/journ al.pone. 0128995

5. O'Hare AM, Tawney K, Bacchetti P, Johansen KL (2003) Decreased survival among sedentary patients undergoing dialysis: results from the dialysis morbidity and mortality study wave 2. Am J Kidney Dis 41(2):447-454. https://doi.org/10.1053/ ajkd.2003.50055

6. Sietsema KE, Amato A, Adler SG, Brass EP (2004) Exercise capacity as a predictor of survival among ambulatory patients with end-stage renal disease. Kidney Int 65(2):719-724. https ://doi.org/10.1111/j.1523-1755.2004.00411.x

7. van Loon IN, Bots ML, Boereboom FTJ, Grooteman MPC, Blankestijn PJ, van den Dorpel MA et al (2017) Quality of life as indicator of poor outcome in hemodialysis: relation with mortality in different age groups. BMC Nephrol 18(1):217. https ://doi.org/10.1186/s12882-017-0621-7

8. Sheng K, Zhang P, Chen L, Cheng J, Wu C, Chen J (2014) Intradialytic exercise in hemodialysis patients: a systematic review and meta-analysis. Am J Nephrol 40(5):478-490. https://doi. org/10.1159/000368722

9. Cheema BS, Singh MA (2005) Exercise training in patients receiving maintenance hemodialysis: a systematic review of clinical trials. Am J Nephrol 25(4):352-364. https://doi. org/10.1159/000087184

10. Chung YC, Yeh ML, Liu YM (2017) Effects of intradialytic exercise on the physical function, depression and quality of life for haemodialysis patients: a systematic review and metaanalysis of randomised controlled trials. J Clin Nurs 26(1314):1801-1813. https://doi.org/10.1111/jocn.13514

11. Smart N, Steele M (2011) Exercise training in haemodialysis patients: a systematic review and meta-analysis. Nephrology (Carlton) 16(7):626-632. https://doi.org/10.111 1/j.1440-1797.2011.01471.x

12. Makhlough A, Ilali E, Mohseni R, Shahmohammadi S (2012) Effect of intradialytic aerobic exercise on serum electrolytes levels in hemodialysis patients. Iran J Kidney Dis 6(2):119-123

13. De Moura Reboredo M, Henrique DMN, De Souza Faria R, Chaoubah A, Bastos MG, De Paula RB (2010) Exercise training during hemodialysis reduces blood pressure and increases physical functioning and quality of life. Artif Org 34(7):586-593. https://doi.org/10.1111/j.1525-1594.2009.00929.x

14. Musavian AS, Soleimani A, Masoudi Alavi N, Baseri A, Savari F (2015) Comparing the effects of active and passive intradialytic pedaling exercises on dialysis efficacy, electrolytes, hemoglobin, hematocrit, blood pressure and health-related quality of life. Nurs Midwifery Stud 4(1):e25922

15. Maheshwari V, Samavedham L, Rangaiah GP (2011) A regional blood flow model for beta2-microglobulin kinetics and for simulating intra-dialytic exercise effect. Ann Biomed Eng 39(12):2879-2890. https://doi.org/10.1007/s10439-011-0383-5

16. Capitanini A, Lange S, D'Alessandro C, Salotti E, Tavolaro A, Baronti ME et al (2014) Dialysis exercise team: the way to sustain exercise programs in hemodialysis patients. Kidney Blood Press Res 39(2-3):129-133. https://doi.org/10.1159/000355787

17. de Souto Barreto P (2017) Exercise for multimorbid patients in primary care: one prescription for All? Sports Med 47(11):21432153. https://doi.org/10.1007/s40279-017-0725-z

18. World Health Organization (2010) Global recommendations on physical activity for health. WHO, Geneva, p 58

19. Tentori F, Blayney MJ, Albert JM, Gillespie BW, Kerr PG, Bommer J et al (2008) Mortality risk for dialysis patients with different levels of serum calcium, phosphorus, and PTH: the Dialysis Outcomes and Practice Patterns Study (DOPPS). Am J Kidney Dis 52(3):519-530. https://doi.org/10.1053/j.ajkd.2008.03.020

20. Moher D, Shamseer L, Clarke M, Ghersi D, Liberati A, Petticrew $M$ et al (2015) Preferred reporting items for systematic review and meta-analysis protocols (PRISMA-P) 2015 statement. Syst Rev 4:1. https://doi.org/10.1186/2046-4053-4-1

21. Cumming G, Fidler F, Kalinowski P, Lai J (2012) The statistical recommendations of the American Psychological Association Publication Manual: effect sizes, confidence intervals, and metaanalysis. Aust J Psychol 64:138-146

22. Jackson D, Turner R (2017) Power analysis for random-effects meta-analysis. Res Synth Methods 8:290-302. https://doi. org/10.1002/jrsm. 1240

23. Cumming G, Calin-Jageman R (2016) Introduction to the new statistics: estimation, open science, and beyond. Routledge, Abingdon

24. Thalheimer W, Cook S (2017) How to calculate effect sizes from published research: a simplified spreadsheet. http://www. uv.es/ friasnav/TEdatospublicados.xls. Accessed 10 Nov 2017

25. Wu Y, He Q, Yin X, He Q, Cao S, Ying G (2014) Effect of individualized exercise during maintenance haemodialysis on exercise capacity and health-related quality of life in patients with uraemia. J Int Med Res 42(3):718-727. https://doi.org/10.1177/03000 60513509037

26. Giannaki CD, Sakkas GK, Karatzaferi C, Hadjigeorgiou GM, Lavdas E, Kyriakides T et al (2013) Effect of exercise training and dopamine agonists in patients with uremic restless legs syndrome: a six-month randomized, partially double-blind, placebocontrolled comparative study. BMC Nephrol 14:194. https://doi. org/10.1186/1471-2369-14-194

27. Dobsak P, Homolka P, Svojanovsky J, Reichertova A, Soucek M, Novakova $M$ et al (2012) Intra-dialytic electrostimulation of leg extensors may improve exercise tolerance and quality of life in hemodialyzed patients. Artif Org 36(1):71-78. https://doi.org/10 $.1111 / \mathrm{j} .1525-1594.2011 .01302 . x$

28. Koh KP, Fassett RG, Sharman JE, Coombes JS, Williams AD (2010) Effect of intradialytic versus home-based aerobic exercise training on physical function and vascular parameters in hemodialysis patients: a randomized pilot study. Am J Kidney Dis 55(1):88-99. https://doi.org/10.1053/j.ajkd.2009.09.025

29. Sakkas GK, Hadjigeorgiou GM, Karatzaferi C, Maridaki MD, Giannaki CD, Mertens PR et al (2008) Intradialytic aerobic exercise training ameliorates symptoms of restless legs syndrome and improves functional capacity in patients on hemodialysis: a pilot study. Asaio J 54(2):185-190. https://doi.org/10.1097/MAT.0b013 e3181641b07

30. Parsons TL, Toffelmire EB, King-VanVlack CE (2004) The effect of an exercise program during hemodialysis on dialysis efficacy, blood pressure and quality of life in end-stage renal disease (ESRD) patients. Clin Nephrol 61(4):261-274 
31. Painter P, Carlson L, Carey S, Paul SM, Myll J (2000) Physical functioning and health-related quality-of-life changes with exercise training in hemodialysis patients. Am J Kidney Dis 35(3):482-492

32. Chigira Y, Oda T, Izumi M, Yoshimura T (2017) Effects of exercise therapy during dialysis for elderly patients undergoing maintenance dialysis. J Phys Therap Sci 29(1):20-23. https://doi. org/10.1589/jpts.29.20

33. Bae YH, Lee SM, Jo JI (2015) Aerobic training during hemodialysis improves body composition, muscle function, physical performance, and quality of life in chronic kidney disease patients. J Phys Therap Sci 27(5):1445-1449. https://doi.org/10.1589/ jpts.27.1445

34. Bohm C, Stewart K, Onyskie-Marcus J, Esliger D, Kriellaars D, Rigatto C (2014) Effects of intradialytic cycling compared with pedometry on physical function in chronic outpatient hemodialysis: a prospective randomized trial. Nephrol Dial Transplant 29(10):1947-1955. https://doi.org/10.1093/ndt/gfu248

35. Golebiowski T, Kusztal M, Weyde W, Dziubek W, Wozniewski M, Madziarska K et al (2012) A program of physical rehabilitation during hemodialysis sessions improves the fitness of dialysis patients. Kidney Blood Pressure Res 35(4):290-296. https://doi. org/10.1159/000335411

36. McMurray A, Blazey L, Fetherston C (2008) The effect of intradialytic foot pedal exercise on blood pressure phosphate removal efficiency and health related quality of life in haemodialysis patients. Renal Soc Aust J 4(2):38-45

37. Parsons TL, Toffelmire EB, King-Van Vlack CE (2006) Exercise training during hemodialysis improves dialysis efficacy and physical performance. Arch Phys Med Rehabil 87(5):680-687

38. Groussard C, Rouchon-Isnard M, Coutard C, Romain F, Malarde L, Lemoine-Morel S (2015) Beneficial effects of an intradialytic cycling training program in patients with end-stage kidney disease. Appl Physiol Nutr Metab 40(6):550-556. https://doi. org/10.1139/apnm-2014-0357

39. Afshar R, Shegarfy L, Shavandi N, Sanavi S (2010) Effects of aerobic exercise and resistance training on lipid profiles and inflammation status in patients on maintenance hemodialysis. Indian J Nephrol 20(4):185-189. https://doi.org/10.4103/0971-4065.73442

40. Wilund KR, Tomayko EJ, Wu PT, Ryong Chung H, Vallurupalli S, Lakshminarayanan B, Fernhall B (2010) Intradialytic exercise training reduces oxidative stress and epicardial fat: a pilot study. Nephrol Dial Transplant 25(8):2695-2701. https://doi. org/10.1093/ndt/gfq106

41. ${ }^{\ddagger}$ Painter P, Carlson L, Carey S, Paul SM, Myll J (2000) Lowfunctioning hemodialysis patients improve with exercise training. Am J Kidney Dis 36(3):600-608. https://doi.org/10.1053/ ajkd.2000.16200

42. Besnier F, Laruelle E, Genestier S, Gie S, Vigneau C, Carre F (2012) Effects of exercise training on ergocycle during hemodialysis in patients with end stage renal disease: Relevance of the anaerobic threshold intensity. [French]. [Effets d'un reentrainement a l'effort sur ergocycle pendant les seances de dialyse chez les insuffisants renaux chroniques hemodialyses: interet d'un travail individualise au premier seuil ventilatoire.]. Nephrologie et Therapeutique 8(4):231-237. https://doi.org/10.1016/j.nephr o.2011.10.002

43. Gomes Neto M, de Lacerda FFR, Lopes AA, Martinez BP, Saquetto MB (2018) Intradialytic exercise training modalities on physical functioning and health-related quality of life in patients undergoing maintenance hemodialysis: systematic review and meta-analysis. Clin Rehabil. https://doi.org/10.1177/0269215518 760380
44. Ketteler M, Block GA, Evenepoel P, Fukagawa M, Herzog CA, McCann L et al (2017) Executive summary of the 2017 KDIGO Chronic Kidney Disease-Mineral and Bone Disorder (CKD-MBD) Guideline Update: what's changed and why it matters. Kidney Int 92:26-36. https://doi.org/10.1016/j.kint.2017.10.001

45. Mohseni R, Emami Zeydi A, Ilali E, Adib-Hajbaghery M, Makhlough A (2013) The effect of intradialytic aerobic exercise on dialysis efficacy in hemodialysis patients: a randomized controlled trial. Oman Med J 28(5):345-349. https://doi.org/10.5001/ omj.2013.99

46. van Vilsteren MC, de Greef MH, Huisman RM (2005) The effects of a low-to-moderate intensity pre-conditioning exercise programme linked with exercise counselling for sedentary haemodialysis patients in The Netherlands: results of a randomized clinical trial. Nephrol Dial Transplant 20(1):141-146. https://doi. org/10.1093/ndt/gfh560

47. Marcelli D, Brand K, Ponce P, Milkowski A, Marelli C, Ok E et al (2016) Longitudinal changes in body composition in patients after initiation of hemodialysis therapy: results from an international cohort. J Ren Nutr 26(2):72-80. https://doi.org/10.1053/j. jrn.2015.10.001

48. Prado CM, Wells JC, Smith SR, Stephan BC, Siervo M (2012) Sarcopenic obesity: a Critical appraisal of the current evidence. Clin Nutr 31(5):583-601. https://doi.org/10.1016/j.clnu.2012.06.010

49. Marcelli D, Usvyat LA, Kotanko P, Bayh I, Canaud B, Etter M et al (2015) Body composition and survival in dialysis patients: results from an international cohort study. Clin J Am Soc Nephrol 10(7):1192-1200. https://doi.org/10.2215/cjn.08550814

50. Souweine JS, Kuster N, Chenine L, Rodriguez A, Patrier L, Morena $\mathrm{M}$ et al (2018) Physical inactivity and protein energy wasting play independent roles in muscle weakness in maintenance haemodialysis patients. PLoS One 13(8):e0200061. https ://doi.org/10.1371/journal.pone.0200061

51. Isoyama N, Qureshi AR, Avesani CM, Lindholm B, Barany P, Heimburger $\mathrm{O}$ et al (2014) Comparative associations of muscle mass and muscle strength with mortality in dialysis patients. Clin J Am Soc Nephrol 9(10):1720-1728. https://doi.org/10.2215/ cjn. 10261013

52. Johansen KL, Painter PL, Sakkas GK, Gordon P, Doyle J, Shubert $\mathrm{T}$ (2006) Effects of resistance exercise training and nandrolone decanoate on body composition and muscle function among patients who receive hemodialysis: a randomized, controlled trial. J Am Soc Nephrol 17(8):2307-2314. https://doi.org/10.1681/ asn.2006010034

53. Longenecker JC, Coresh J, Powe NR, Levey AS, Fink NE, Martin A et al (2002) Traditional cardiovascular disease risk factors in dialysis patients compared with the general population: the CHOICE Study. J Am Soc Nephrol 13(7):1918-1927

54. Anderson L, Brown JP, Clark AM, Dalal H, Rossau HK, Bridges C, Taylor RS (2017) Patient education in the management of coronary heart disease. Cochrane Database Syst Rev 6:CD008895. https://doi.org/10.1002/14651858.CD008895.pub3

55. Kim Y, Evangelista LS, Park YG (2015) Anxiolytic effects of music interventions in patients receiving incenter hemodialysis: a systematic review and meta-analysis. Nephrol Nurs J 42(4):339_ 347 (quiz 348)

56. Koca Kutlu A, Eren AG (2014) Effects of music on complications during hemodialysis for chronic renal failure patients. Hemodial Int 18(4):777-784. https://doi.org/10.1111/hdi.12161

57. Burrai F, Lupi R, Luppi M, Micheluzzi V, Donati G, Lamanna G et al (2018) Effects of listening to live singing in patients undergoing hemodialysis: a randomized controlled crossover study. Biol Res Nurs. https://doi.org/10.1177/1099800418802638 